\title{
First Year of Observations with SOHO/EIT of the "Quiet" Sun Corona
}

\author{
F. Portier-Fozzani, A. J. Maucherat and the EIT Team \\ Laboratoire d'Astronomie Spatiale, CNRS, BP8, 13376 Marseille Cedex \\ 12, France
}

\begin{abstract}
Since January 1996 (EIT first light) the Extreme Ultraviolet Telescope aboard SOHO has produced about 20,000 wide-field images of the corona and transition regions. Four different emission lines ( $\mathrm{He} \mathrm{II,} \mathrm{Fe}$ IX/X, Fe XII, Fe XV) were selected to detail morphologies of magnetic structures in the corona. They show the different structures present in the corona with information about their topologies (Neupert et al. 1998). They provide the global temperature distribution in the quiet corona in the range 0.5 to $3 \times 10^{6} \mathrm{~K}$.

The evolution of the corona during the first year of the SOHO mission revealed its nonuniform aspect and the nonregularity of the appearance of new active regions. Changes observed in active regions and coronal holes (e.g., August-September 1996) showed the complex role of magnetic fields including magnetic interactions and possible reconnections needed to explain some loop morphology evolution.
\end{abstract}

\section{Introduction}

A full description of the EIT Telescope aboard SOHO can be found in Delaboudiniere et al. (1995). Since February 1996 at least one image per day in all 4 wavelengths of the whole Sun have been taken (except during CCD bakeout done to increase efficiency). Movies were made on smaller areas to give shorter time evolution. The satellite observes the Sun 24 hours per day but, due to telemetry sharing, precise targets have to be chosen.

The Sun was at the minimum level of the 11 year cycle during 1996, but even at that time, some activity was present. Comparison between the amount of activity (determined by active region counts) with time and the sunspot number shows the well known links via magnetic fields. We followed the evolution with studies of 3D coronal structures (Portier-Fozzani et al. 1996).

\section{1996: A Full Year of Observations of the Quiet Sun's Corona}

During January 1996 three Active Regions (ARs) were present. There was also another active fuzzy area, in which two new ARs appeared the next month. A decrease of activity was noted in March with only two large and two small ARs.

The lowest activity occurred in April with 4 small ARs: the "Quiet" Sun's 


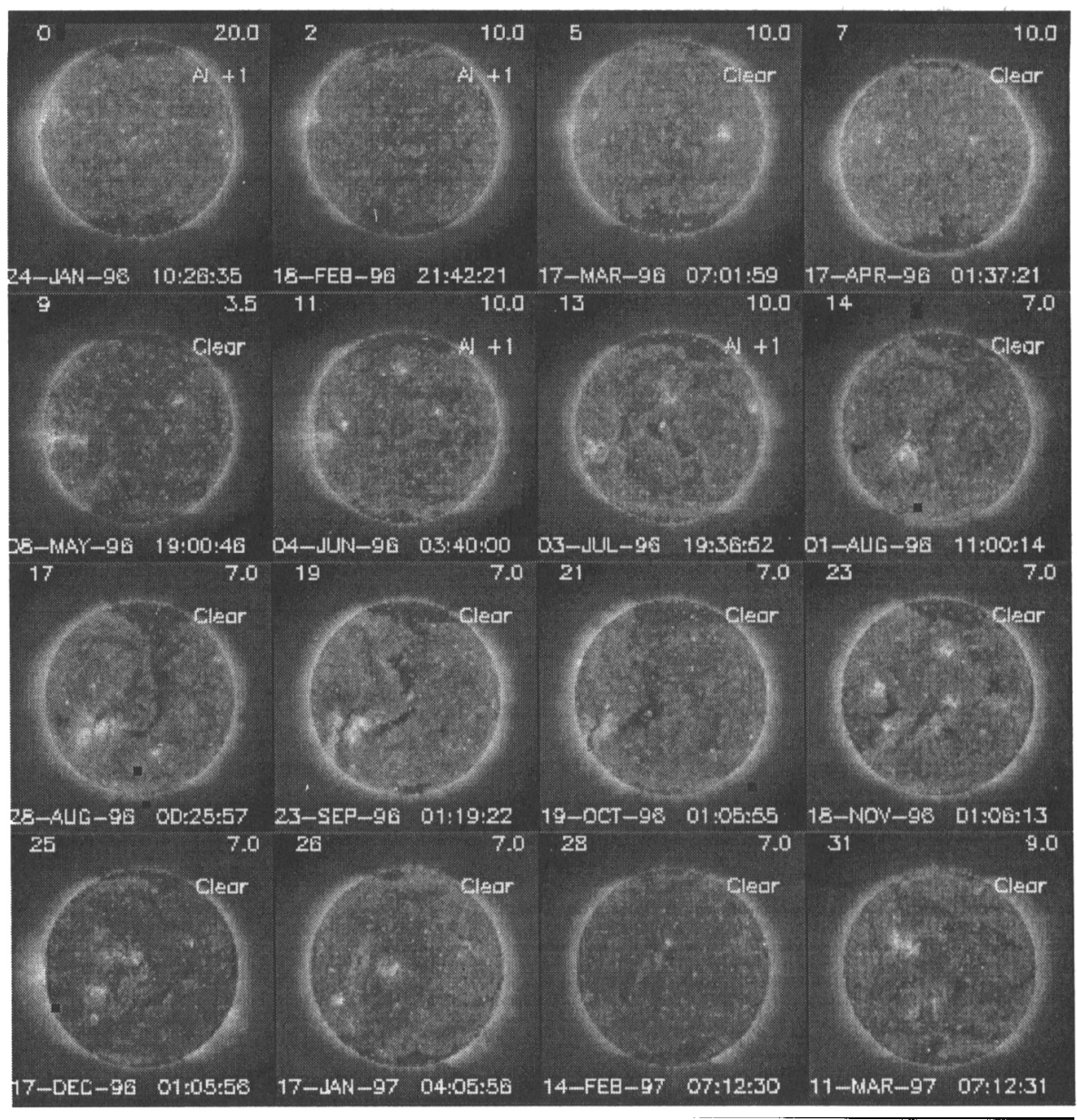

Figure 1. Activity of the corona observed with SOHO EIT in FeXII at $195 \AA$ during the January 1996-March 1997 period. 
corona remained in fact still active. During May, two large ARs were present, both shared and spread in four parts in June. July showed an increase of activity. ARs and Coronal Holes ( $\mathrm{CHs}$ ) became more frequent, introducing strong nonuniformity in the corona. An interaction between $\mathrm{CHs}$ and ARs began in August. A strong Equatorial Coronal Hole (ECH) developed from the North polar $\mathrm{CH}$ toward an AR loop present in the southern hemisphere (Figure 1). Then, in September, the morphology of the AR changed completely, with fewer loops inside. By October, the ECH had decreased. In November, 5 ARs were present and 7 in December, showing the increase of activity of the cycle.

$K$. Harvey (1997, private communication) found that the minimum of the sunspot number was in May 1996, but the appearance of the new cycle activity was unusual. From EIT results, the AR number was minimum around April 1996 (Portier-Fozzani 1997). On the other hand, the main presence of a large $\mathrm{CH}$ suggests that the minimum of coronal activity occurred during June-October.

\section{Global Evolution of the Coronal Activity}

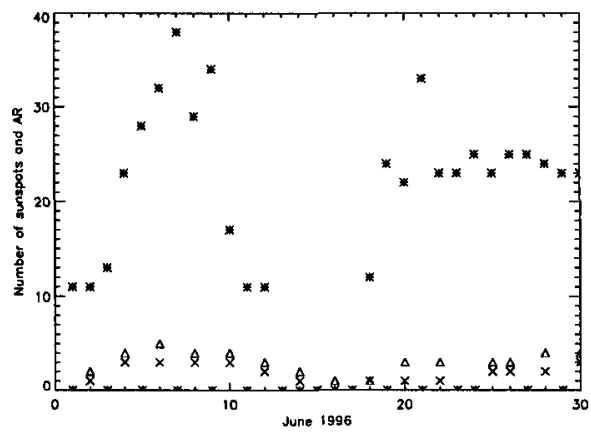

Figure 2. Coronal and photospheric activity during June 1996: (* represents the sunspot number, $\triangle$ the $A R$ number and $X$ the number of big ARs)

During that minimum period the corona appeared very nonuniform. An example is given by mid-February when the Sun had an active region on one side while the other side had nothing. Apparitions of AR were nonlinear with time; sometimes 1,2 or 3 could appear during a short time like in October-December.

Phillips (1995) mentioned that ARs do not always correspond to sunspots despite the expected link via the magnetic field. This is mainly the case in 1996, except for the weak activity in June 1996 (Figure 2), where coronal numbers were proportional to sunspot numbers. The reason is probably that only a part of the sunspots influence strongly coronal activity and are able to build via the magnetic field some loops with high enough temperature to be seen at all wavelengths.

The number of large active regions seen on the disk are proportional to the absolute value of the magnetic field. This was expected because the plasma is frozen to the strong magnetic field and then creates large ARs (Klimchuk et al. 1998). Finally, by studying $C, M, X$ flare eruptions, no observed links are noted between the numbers of $A$ Rs and flares. As there was no relation with 
the time of the flares, this activity could be linked to AR's stability and not to their statistical numbers (Portier-Fozzani and Noens 1997).

\section{Conclusions}

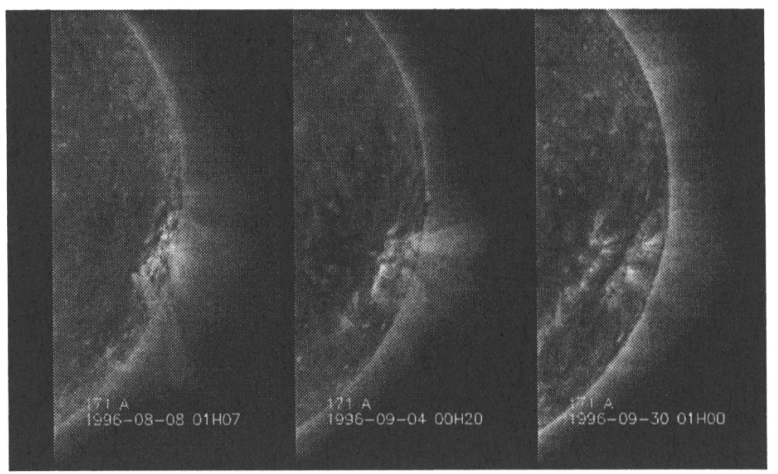

Figure 3. Loop evolution in $\mathrm{Fe} \mathrm{IX} / \mathrm{X}$

Magnetic fields drive coronal evolution. Synoptic EIT observations give us the possibility to study the global change of the corona with time. Comparison with other observations help us to understand the relationship between different physical processes present in the Sun at the minimum of the cycle. The evolution of the corona, such as changes in AR loop morphology (e.g., Figure 3), recall the role of different magnetic fields and possible reconnections (Portier-Fozzani et al. 1998). Thanks to a high telemetry rate, the birth of a CME was recorded on December 24, 1996 (Moses et al. 1998). In conclusion, observations will certainly yield more and more surprises as the activity cycle grows toward maximum, but even at the minimum lots of events occurred in the corona.

\section{References}

Delaboudiniere, J.-P. et al. Solar Phys., 162, 291

Klimchuk, J., D. Moses and F. Portier-Fozzani 1998, ApJ, submitted

Portier-Fozzani, F. et al. 1996, in Magnetic Reconnection in the Solar Atmosphere, (eds.) R. Bentley and J. Mariska, San Francisco, p. 402

Portier-Fozzani, F. and J.C. Noens 1997, in Science with Themis, Publication of Paris-Meudon Observatory, p. 281

Portier-Fozzani, F. June 1997, LAS Internal Report

Portier-Fozzani, F. 1998, A\&A, submitted

Phillips, K. 1995, Guide to the Sun, Cambridge University Press

Neupert, W.M. et al. 1998, JGR, in press

Moses, D. et al. 1998, Solar Phys., submitted 